\section{Brain, Behavior and Evolution}

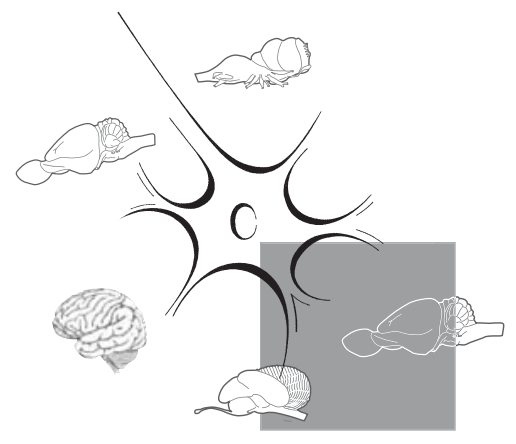

Evolution operates through intraspecific variation. It takes the genetic variation responsible for the differences that we see between individuals and drives them to fixation, whether by chance or selection, and leads to the emergence of new species. This understanding drives much of our current thinking of biology and underlies the statistical tests of selective pressures. It allows us to better understand the processes that occurred long ago, in evolutionary time, to create the diverse array of species and phenotypes that we see around us today.

When we do comparative studies of the brain today, we are attempting to reconstruct evolutionary changes long since fixed. This can be accomplished by focusing on the genetic differences between species, but our methodologies, especially outside of protein-coding regions, are still in their infancies and do not currently achieve sufficient statistical power. Besides, attributing specific functional effects to fixed changes can be daunting even if they are successfully identified. By focusing on the phenotype, the ultimate realization of the genetic change, comparative neuroanatomists can inform on the underlying mechanisms that created these differences between species. Two schools of thought have predominated for our understanding of brain evolution, focusing mainly on the relationship between regions of the brain and

\title{
How Brains Are Built: Genetics and Evolution
}

\author{
Eric J. Vallender \\ New England Primate Research Center, Harvard Medical School, Southborough, Mass., USA
}

to what degree they coevolve. While existing on a continuum, the mosaic approach favors the idea that brains consist of a large number of independently evolving modules; the concerted evolution approach envisions just a few. Originating in comparative studies across species, these hypotheses have important implications for the molecular evolution of neurodevelopmental programs.

The two competing hypotheses derived largely from comparative analysis of brain size between species using the same data sets [Stephan et al., 1981]. Through one lens emerged the concerted program, which identifies two principal modules, the first consisting mainly of the neocortex and the second of the limbic system and olfactory bulb [Finlay and Darlington, 1995]. Through the second lens, size change was seen as independent in at least five distinct functional systems (subject to data availability): the hippocampal formation, amygdala, vestibular system, visual/auditory sensory system, and olfactory bulb [Barton and Harvey, 2000]. These discussions, including arguments for and against, have been litigated and relitigated [for example, see Finlay et al., 2001 and associated commentary]; there is little to be added here on that front.

Conceptually, it is easy to predict how altering an underlying developmental pro- gram might lead to a coordinated change across multiple brain regions. A general extension of neurogenesis, for example, would lead to larger sizes across regions proportional to their neuronal density. The same could be said for accelerated cell cycling or for changes in the numbers of neuronal stem cells either through their birth or death. In each of these cases one relatively simple change would lead to a widespread effect. However, this pleiotropy has been a major argument against concerted effects generally. An early upstream change can dramatically affect the system, but often in problematic and unanticipated ways, and it is more likely to 'break' it than a smaller, downstream change.

The mosaic approach to brain variation and evolution is much more amenable to our engineer's minds. Parts are more easily swapped in and out independently, finding the perfect size and fit for a particular ecological niche or species need. Certainly a designer would take this approach. However, it is trickier to do. Changes would have to be localized and would have to have their effects insulated from other regions. It is not impossible to conceive of mechanisms by which this might occur, but it requires the introduction of greatly more complexity. Luckily, though, the brain does not lack for genetic complexity, and if evolution has taught us anything it is that

\section{KARGER}

E-Mail karger@karger.com

www.karger.com/bbe
(C) 2013 S. Karger AG, Basel

0006-8977/13/0812-0071\$38.00/0
Eric J. Vallender

New England Primate Research Center

Harvard Medical School, One Pine Hill Drive

Southborough MA 01772 (USA)

E-Mail eric_vallender@hms.harvard.edu 
where there is a strong enough need nature will find a way.

While we may be interested primarily in how differences in brains arise between species, it is extremely useful to consider intraspecific variation. Ultimately, interspecific divergence is derived from intraspecific variation; and while it is true that extant variation is not necessarily representative of the long vanished variability that led to species differences, the breadth of extant variation is perhaps representative of the opportunities of which evolution could avail itself. Further, identifying the molecular underpinnings of brain size, change is much more tractable when we look at intraspecific variation. Tools for quantitative trait analysis are developed and robust, while studies of molecular evolution are necessarily more confounding and often correlative at best.

It is unsurprising then that the question of developmental programs has now moved into an intraspecific arena. Recently, quantitative trait loci (QTL) studies in mice identified specific gene regions associated with weight or volume of seven brain regions as well as overall brain and body weight [Hager et al., 2012]. These studies used a panel of recombinant inbred mouse lines derived from parental C57BL/6J and DBA/2J mice (BXD) [Peirce et al., 2004]. By comparing these inbred lines for phenotypic differences, in this case for brain region sizes, it is then possible to correlate the changes with genomic regions inherited from one of the two parental strains. This is a powerful experimental system for detecting genetic causes of numerous phenotypes, though it is obviously limited to those variants present in the original parental strains. However, this approach is truly genomic and does not rely on any a priori hypothesis as to what researchers should expect, whether specific candidate genes or specific types of changes. This latter is important because it offers a way to identify changes in regulatory regions that may have effects, even in the absence of those regions being recognized as functional. The recent work identifies one to three loci affecting each of seven defined subregions as well as overall brain weight [Hager et al., 2012]. It further identifies two situations of epistasis where variants at two distinct locations come together to affect phenotypic change in concert. While further work will be required to identify the specific changes responsible for the phenotype, and indeed the specific functional ele- ment that these changes reside in, they represent some of the first examples of the transition of the field from phenotype to genotype.

The mouse studies offer a powerful experimental system and this QTL work is unlikely to be possible elsewhere. However, the inbred lines that make these studies feasible must also necessarily limit the universe of variability considered. Human studies, and indeed almost all studies of outbred populations, capture a much greater amount of genetic variation but require substantially greater sample sizes to have sufficient statistical power. However, by focusing on phenotypic variation, it is possible to infer underlying genetic structure even if specific genes cannot be identified. In both humans [Charvet et al., 2013] and nonhuman mammals [Finlay et al., 2011], studies of phenotypic variation between individuals parallel the fixed differences seen between species using the methodologies reducing variability to two factors. These approaches ask how phenotypic variability exists within a population, whether regions covary or exist independently. While concerted phenotypic variation does not necessarily have to arise from single loci, independent phenotypic variation must result from independent genetic variation at some point. In the human study, observed phenotypic variation is largely concerted across regions save for an independently varying component loaded on the limbic system [Charvet et al., 2013]. The implication of this finding is that the dominant, if not only, genetic variation affecting human brain size and structure is likely operating on these more global levels.

Human studies of genetic variation affecting the normal range of brain sizes are still largely absent from the literature, but there is a larger literature focusing on the numerous pathogenic cephalic disorders [Pang et al., 2008]. Notable in this is that pathogenesis tends to affect the whole of the brain rather than only specific regions and that specific genetic mutations have been identified. However, it is less clear what the general lack of pathogenesis focused on the growth of specific brain regions means. It is possible that this reflects a lack of a developmental program upon which to act, but it is also possible that these genes are simply less likely to be pathogenic, whether through their location within developmental pathways or through redundancy and effect size. It is also perhaps notable that while studies of knockout mice can and do recapitulate the human cephalic pathologies, the recent QTL studies by and large find little natural variation affecting brain size as a whole [Hager et al., 2012].

The findings from human cephalic disorders and from the intraspecies studies perhaps offer a clue as to the genetic underpinnings of change. Changes in proteins are often very large and wide-reaching. Indeed, most changes resulting in microcephaly, macrocephaly and lissencephaly affect proteins. These same changes are perhaps less likely to affect specific regions exclusively and independently of others. Rather, changes affecting only specific regions could more likely be found in regulatory regions where perturbations to spatial or temporal expression patterns or intensity can have major influences that remain discrete. While the role of regulatory change in producing dramatic phenotypic change, particularly in humans, has long been recognized [King and Wilson, 1975], detecting this change at the genetic or genomic level has been more difficult. Interspecific studies of molecular evolution have been much more successful at identifying selection in protein coding regions even when regulatory domains are known. Intraspecific studies are much more agnostic to functional category. Still, we do not know for certain whether extant variation reflects that which ultimately led to evolutionary divergence, and polymorphismbased genetic and genomic studies can detect only the former, not the latter.

Another question arises regarding the relative role of adaptive or 'near-neutral' evolution. While it is certainly comforting to believe that human brain expansion was evolutionarily adaptive, the same likely cannot be said for all brain size differences between species. Similarly amongst extant populations within a species, even humans, it certainly is not clear that phenotypic variation in brain size is adaptive one way or another. One potentially intriguing possibility is that neutral evolution changes the brain largely as a single unit, but that selective forces shape the brain primarily in a 'mosaic' fashion. This is perhaps supported by the fact that the olfactory bulb, traditionally argued to be one of the brain regions most sensitive to adaptive natural selection, emerges as an independent element across all frameworks. This may also be reflected in the pathogenesis of cephalic disorders in humans where most variation in individuals affects the whole brain, 
but radical change is most likely detrimental.

Finally, it is worth touching upon the larger picture. Changes in the brain during evolution are particularly interesting because of the cognitive and behavioral phenotypes with which it presumably correlates but whose evolution is difficult to discern. The study of brain evolution is a useful tool because brains are relatively big, physical, things that we can see in the fossil record, at least as endocasts, and whose evolution is more clear. And while certain aspects of behavior are obviously correlat- ed with the size of the brain or of specific regions (one thinks of the relative size of the olfactory bulb and its relation to sensory perception or perhaps the association between executive function and the prefrontal cortex), other aspects are much more subtle and may be modulated through connectivity, neurochemical modulation, or other factors that may not translate as directly to sizes and shapes. Of course, one may question whether the behaviors thus affected are often drivers of speciation.

Neither genes nor phenotypes exist in isolation; there is a complex interplay across both modalities as well as between them. Gene networks are complex systems where alterations in multiple places can result ultimately in similar effects. Nowhere is this more true than in the brain. There is clearly more work to be done in this arena, but our understanding of the brain in an evolutionary developmental context will not only illuminate the evolution of species-specific behaviors and adaptation, but will also elucidate the complex interplay between mutation and selection in gene networks.

\section{References}

Barton RA, Harvey PH (2000): Mosaic evolution of brain structure in mammals. Nature 405: 1055-1058.

-Charvet CJ, Darlington RB, Finlay BL (2013): Variation in human brains may facilitate evolutionary change toward a limited range of phenotypes. Brain Behav Evol 81:74-85.

Finlay BL, Darlington RB (1995): Linked regularities in the development and evolution of mammalian brains. Science 268:1578-1584.
Finlay BL, Darlington RB, Nicastro N (2001): Developmental structure in brain evolution. Behav Brain Sci 24:263-278, discussion 278308.

Finlay BL, Hinz F, Darlington RB (2011): Mapping behavioural evolution onto brain evolution: the strategic roles of conserved organization in individuals and species. Philos Trans R Soc Lond B Biol Sci 366:2111-2123.

Hager R, Lu L, Rosen GD, Williams RW (2012): Genetic architecture supports mosaic brain evolution and independent brain-body size regulation. Nat Commun 3:1079.

King MC, Wilson AC (1975): Evolution at two levels in humans and chimpanzees. Science 188:107-116.
Pang T, Atefy R, Sheen V (2008): Malformations of cortical development. Neurologist 14:181191.

Peirce JL, Lu L, Gu J, Silver LM, Williams RW (2004): A new set of BXD recombinant inbred lines from advanced intercross populations in mice. BMC Genet 5:7.

Stephan H, Frahm H, Baron G (1981): New and revised data on volumes of brain structures in insectivores and primates. Folia Primatol (Basel) $35: 1-29$. 